Pacific Journal of Mathematics

WEAK DENSENESS OF MONATOMIC MEASURES ON 


\title{
WEAK DENSENESS OF NONATOMIC MEASURES ON PERFECT, LOCALLY COMPACT SPACES
}

\author{
NORMAN Y. LUTHER
}

\begin{abstract}
Our primary result is that the space of all compact zeroset-regular, nonatomic, countably additive Baire measures is dense, with respect to the weak topology, in the space of all finitely additive, zero-set regular Baire measures if the underlying topological space is locally compact, Hausdorff, and perfect. Moreover, a corresponding result holds for Borel measures. These results yield, as easy corollaries, the existence of nonzero, nonatomic, countably additive, compactregular Baire and Borel measures on a locally compact, Hausdorff space which contains a nonempty perfect subset. Two converses conclude the paper.
\end{abstract}

We use the methods and results of Knowles [5] and Varadarajan [10] to prove our primary result (Theorem 3.1). This result extends an earlier result in which the underlying space was assumed compact, Hausdorff, and perfect (see [5, Th. 1 (i) and Remark (i), p. 65] and $[2$, p. 214]).

2. Preliminaries. Let $X$ be a Hausdorff topological space. If $Y$ is a subspace of $X$, we use $\mathfrak{F}(Y)$ and $\mathfrak{B}(Y)$ to denote the algebra and $\sigma$-algebra, respectively, generated by the zero-sets of $Y$; we let $\mathfrak{F}^{*}(Y)$ and $\mathfrak{B}^{*}(Y)$ denote the algebra and $\sigma$-algebra, respectively, generated by the closed subsets of $Y . \mathfrak{B}(Y)$ and $\mathfrak{B}^{*}(Y)$ are called the Baire and Borel subsets of $Y$, respectively. For brevity, we let $\widetilde{F}=$ $\mathfrak{F}(X), \mathfrak{B}=\mathfrak{B}(X), \mathfrak{F}^{*}=\mathfrak{F}^{*}(X)$, and $\mathfrak{B}^{*}=\mathfrak{B}^{*}(X)$. A measure on an algebra $\mathfrak{A}$ is a nonnegative, finite, finitely additive set function on $\mathfrak{A}$. $A$ signed measure on $\mathfrak{U}$ is the difference of two measures on $\mathfrak{A}$. Measures on $\mathfrak{B}(Y)$ and $\mathfrak{B}^{*}(Y)$ will be called Baire and Borel measures on $Y$, respectively. We use $\mathfrak{S}(Y), \mathfrak{C}(Y), \mathfrak{c}_{0}(Y)$ and $\mathfrak{Z}(Y)$ to denote the classes of closed sets, compact sets, compact zero-sets and zerosets of $Y$, respectively. For brevity, we let $\mathfrak{S}=\mathscr{S}_{\mathfrak{C}}(X), \quad \mathfrak{S}=\mathbb{C}(X)$, $\mathfrak{c}_{0}=\mathfrak{c}_{0}(X)$ and $3=3(X)$.

If $\mathfrak{U}$ is an algebra of sets and $\mathfrak{D} \subset \mathfrak{A}$, a measure $m$ on $\mathfrak{U}$ shall be called $\mathfrak{D}$-regular in case

$$
m(A)=\sup \{m(D) ; A \supset D \in \mathfrak{D}\} \text { for every } A \in \mathfrak{X} \text {. }
$$

Characteristically, $\left(\mathcal{D}\right.$ will play the role of $\mathfrak{S}_{\mathfrak{C}}, \mathfrak{E}, \mathfrak{S}_{0}$, or 3 .

Let $m$ be a measure on an algebra $\mathfrak{A}$. A set $E \in \mathfrak{X}$ is an atom for $m$ in case (i) $m(E)>0$ and (ii) for every $F \subset E$ for which $F \in \mathfrak{A}$ either $m(F)=0$ or $m(E-F)=0 . \quad m$ is called nonatomic in 
case it has no atoms. We shall need the following special case of [4, Th. 2.4].

Lemma 2.1. Let $\mu, \nu$ be countably additive measures on a $\sigma$ algebra $\mathscr{S}$ and suppose $\nu \leqq \mu$. If $\mu$ is nonatomic, then so is $\nu$.

Incidentally, one cannot replace " $\sigma$-algebra" by "algebra" in the preceding result.

Finally, if $\mathfrak{A}$ is an algebra and $\mathfrak{D} \subset \mathfrak{A}$, we let

$\mathfrak{M}^{+}(\mathfrak{A}, \mathfrak{D})=\{m ; m$ is a $\mathfrak{D}$-regular measure on $\mathfrak{A}\}$

$\mathfrak{M}(\mathfrak{A}, \mathfrak{D})=\left\{m_{1}-m_{2} ; m_{1}, m_{2} \in \mathfrak{M}^{+}(\mathfrak{U}, \mathfrak{D})\right\}$

$\mathfrak{R}^{+}(\mathfrak{A}, \mathfrak{D})=\{m ; m$ is a $\mathfrak{D}$-regular, countably additive, nonatomic measure on $\mathfrak{A}\}$

$$
\mathfrak{R}(\mathfrak{U}, \mathfrak{D})=\left\{m_{1}-m_{2} ; m_{1} m_{2} \in \mathfrak{N}^{+}(\mathfrak{A}, \mathfrak{D})\right\} \text {. }
$$

We note that $\mathfrak{M}(\mathfrak{A}, \mathfrak{D})$ and $\mathfrak{R}(\mathfrak{U}, \mathfrak{D})$ are linear spaces whenever $\mathfrak{D}$ is closed under finite unions, which will be the case in all of our considerations. In order to relate our notation with that of Varadarajan [10], we remark that our $\mathfrak{M}(\mathfrak{F}, 3)$ is precisely Varadarajan's $\mathfrak{M}(X)$ [10, p. 164] and that $\mathfrak{N}\left(\mathfrak{F}, \mathfrak{S}_{0}\right) \subset \mathfrak{M}\left(\mathfrak{F}, \mathfrak{S}_{0}\right) \subset \mathfrak{M}_{t}(X)$ [10, Th. 29, p. 179] where $\mathfrak{M}_{t}(X)$ denotes the space of tight signed measures [10, p. 174].

We shall consider $\mathfrak{M}(\mathfrak{A}, \mathfrak{D})$ primarily when $(\mathfrak{U}, \mathfrak{D})$ is one of the

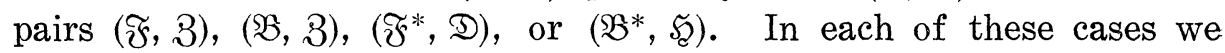
put the weak topology (called the weak*-topology by some authors) on $\mathfrak{M}(\mathfrak{A}, \mathfrak{D})$. This is the topology with basic neighborhoods of the form

$$
N\left(m_{0} ; A, \varepsilon\right)=\left\{m \in \mathfrak{M}(\mathfrak{A}, \mathfrak{D}) ;\left|\int f d m-\int f d m_{0}\right|<\varepsilon \text { for every } f \in A\right\}
$$

where $m_{0} \in \mathfrak{M}(\mathfrak{A}, \mathfrak{D}), \varepsilon>0$, and $A$ is a finite subset of $C^{*}(X)$, the set of all bounded, continuous functions on $X$. $\mathfrak{M}(\mathfrak{A}, \mathfrak{D})$ will be completely

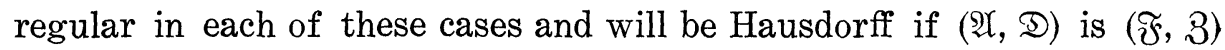
or $(\mathfrak{B}, \mathfrak{3})$; $\mathfrak{M}\left(\mathfrak{F}^{*}, \mathfrak{K}\right)$ and $\mathfrak{M}\left(\mathfrak{B}^{*}, \mathfrak{K}\right)$ are Hausdorff if $X$ is normal (see Taylor [9, pp. 151-153] and the proof of [10, Th. 1, p. 181]). It may be relevant to Theorem 3.1 to point out that the linear subspaces $\mathfrak{M}\left(\mathfrak{F}^{*}, \mathfrak{S}\right)$ and $\mathfrak{M}\left(\mathfrak{B}^{*}\right.$, (ㄷ) of $\mathfrak{M}\left(\mathfrak{F}^{*}, \mathfrak{F}\right)$ and $\mathfrak{M}\left(\mathfrak{B}^{*}, \mathfrak{S}\right)$, respectively, are Hausdorff in the weak topology if $X$ is completely regular, hence also if $X$ is locally compact, Hausdorff. Finally, we note that a net $\left\{m_{\alpha}\right\} \subset \mathfrak{M}(\mathfrak{A}, \mathfrak{D})$ converges to $m \in \mathfrak{M}(\mathfrak{U}, \mathfrak{D})$ in the weak topology if, and only if,

$$
\int f d m_{\alpha} \rightarrow \int f d m \text { for every } f \in C^{*}(X) .
$$

If $m$ is a signed measure on an algebra $\mathfrak{A}$, we use $m^{+}$and $m^{-}$ to denote its positive and negative variations, respectively. If $m=$ 
$m_{1}-m_{2}$ where $m_{1}, m_{2}$ are measures on $\mathfrak{A}$, then $m^{+} \leqq m_{1}$ and $m^{-} \leqq m_{2}$. Thus clearly we have $m \in \mathfrak{M}(\mathfrak{H}, \mathfrak{D})$ if, and only if, $m^{+}, m^{-} \in \mathfrak{M}^{+}(\mathfrak{A}, \mathfrak{D})$. Moreover, we have

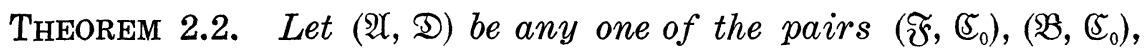
$\left(\mathfrak{F}^{*}, \mathfrak{E}\right)$, or $\left(\mathfrak{B}^{*}, \mathfrak{S}\right)$. Then $m \in \mathfrak{N}(\mathfrak{N}, \mathfrak{D})$ if and only if, $m^{+}, m^{-} \epsilon$ $\mathfrak{N}^{+}(\mathfrak{A}, \mathfrak{D})$.

The following two lemmas, which will also have further application later, will be helpful in the proof of Theorem 2.2.

LEMMA 2.3. Suppose $m$ is a countably additive measure on an algebra $\mathfrak{A}$ and let $\mu$ be the unique extension of $m$ to a countably additive measure on the $\sigma$-algebra generated by $\mathfrak{A}$. If $\mu$ is nonatomic, then so is $m$.

The proof is fairly routine and will be left to the reader.

The converse of the preceding lemma is not true in general, but is true with the added assumptions that are inherent in our setting. This fact is contained in the following lemma.

LEMMA 2.4. Let $X$ be a topological space and let $m$ be a measure on $\mathfrak{F}=\mathfrak{F}(X)$ [resp., $\mathfrak{F}^{*}=\mathfrak{F}^{*}(X)$ ] which is countably additive. Let $\mu$ denote the unique extension of $m$ to a countably additive measure on $\mathfrak{B}=\mathfrak{B}(X)$ [resp., $\left.\mathfrak{B}^{*}=\mathfrak{B}^{*}(X)\right]$.

(i) $m$ is $\mathbb{E}_{0}$-regular [resp., (5-regular], if and only if, $\mu$ is.

(ii) Suppose $m$ is 3-regular [resp., $\mathfrak{S}$-regular]. Then $m$ is nonatomic if, and only if, $\mu$ is.

REMARK. With respect to (ii), it should be noted that every countably additive measure on $\mathfrak{F}$ (or on $\mathfrak{B}$ ) is necessarily 3-regular [10, Th. 18, p. 171].

Proof. (i) The one implication is clear. Suppose $m$ is $\mathbb{F}_{0}$-regular [resp., (5-regular]. Then $\mu$ is 3-regular [resp., $\mathfrak{S}$-regular] by a standard proof ([10, Th. 18, p. 171]). Let $E \in \mathfrak{B}$ [resp., $\left.\mathfrak{B}^{*}\right]$ and $\varepsilon>0$. There is a set $F \in \mathbb{Z}$ [resp., $\mathfrak{S}$ ] such that $F \subset E$ and $\mu(E-F)<\varepsilon / 2$. There exists $C \in \mathfrak{E}_{0}$ [resp., (5] such that $\mu(X-C)=m(X-C)<\varepsilon / 2$. Let $H=F \cap C$. Then $H \in \mathfrak{E}_{0}$ [resp., (5)], $H \subset E$, and $\mu(E-H)<\varepsilon$.

(ii) The one implication follows from Lemma 2.3. To prove the other implication, we note once again that $\mu$ is 3-regular [resp., $\mathfrak{S}$ regular]. Hence if $\mu$ has an atom, it must have an atom $Z \in \mathbb{Z}$ 
[resp., $\mathfrak{S}$ ]. But then $Z$ would also be an atom for $m$ which is a contradiction.

Proof of Theorem 2.2. The only nontrivial part of the proof is showing that $m \in \mathfrak{N}(\mathfrak{A}, \mathfrak{D})$ implies that $m^{+}, m^{-}$are nonatomic. This follows directly from Lemma 2.1 in case $(\mathfrak{A}, \mathfrak{D})$ is $\left(\mathfrak{B}, \mathfrak{S}_{0}\right)$ or $\left(\mathfrak{B}^{*}, \mathfrak{F}\right)$.

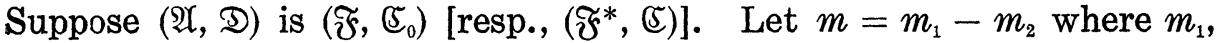
$m_{2} \in \mathfrak{N}^{+}(\mathfrak{H}, \mathfrak{D})$. Then $m^{+} \leqq m_{1}$ and $m^{-} \leqq m_{2}$ so that $m^{+}, m^{-}$are 3regular [resp., SE-regular]. Let $\mu_{1}, \mu_{2}$ denote the unique extensions of $m_{1}, m_{2}$, respectively, to countably additive measures on $\mathfrak{B}$ [resp., $\mathfrak{B} *$ ]. Then $\mu_{1}, \mu_{2}$ are nonatomic by Lemma 2.4 (ii). Now $m^{+}, m^{-}$are countably additive. Let $\nu_{1}, \nu_{2}$ denote the unique extensions of $m^{+}, m^{-}$, respectively, to countably additive measures on $\mathfrak{B}$ [resp., $\mathfrak{B}^{*}$ ]. Then $\nu_{1} \leqq \mu_{1}$ and $\nu_{2} \leqq \mu_{2}$ because $m^{+} \leqq m_{1}$ and $m^{-} \leqq m_{2}$. Thus $\nu_{1}, \nu_{2}$ are nonatomic by Lemma 2.1. Consequently, $\mathrm{m}^{+}, \mathrm{m}^{-}$are nonatomic by Lemma 2.4 (ii).

3. Main result. Let $X$ be a topological space. We say that a nonempty subset $Y$ of $X$ is perfect in case $Y$ contains no isolated points (with respect to the induced topology on $Y$ ).

THEOREM 3.1. Let $X$ be a perfect, locally compact, Hausdorff space.

(i) $\mathfrak{N}\left(\mathfrak{F}, \mathfrak{S}_{0}\right)$ is dense in $\mathfrak{M}(\mathfrak{F}, 3)$ with the weak topology.

(ii) $\mathfrak{R}\left(\mathfrak{B}, \mathfrak{C}_{0}\right)$ is dense in $\mathfrak{M}(\mathfrak{B}, 3)$ with the weak topology.

(iii) $\mathfrak{N}\left(\mathfrak{F}^{*}, \mathfrak{S}\right)$ is dense in $\mathfrak{M}\left(\mathfrak{F}^{*}, \mathfrak{F}\right)$ with the weak topology.

(iv) $\mathfrak{R}\left(\mathfrak{B}^{*}, \mathfrak{C}\right)$ is dense in $\mathfrak{M}\left(\mathfrak{B}^{*}, \mathfrak{S}\right)$ with the weak topology.

REMARK. In the setting of Varadarajan [10], ( $i)$ says that the set of all differences of $\mathbb{S}_{0}$-regular, countably additive, nonatomic measures on $\mathfrak{F}$ is dense in $\mathfrak{M}(X)$.

The proof of the preceding theorem will require the following three lemmas.

Lemma 3.2. Let $X$ be a topological space.

(i) If $X$ is perfect, then every open subset of $X$ is perfect.

(ii) If $Y$ is a perfect subset of $X$, then the closure (in $X)$ of $Y$ is perfect.

The proof is easy and will be omitted.

If $\mathfrak{X}$ is an algebra of subsets of $X$ and $x \in X$, we shall use $p_{x}$ to denote the measure on $\mathfrak{A}$ defined by 


$$
p_{x}(E)=\left\{\begin{array}{ll}
1 & \text { if } x \in E \\
0 & \text { if } x \notin E
\end{array} \text { for every } E \in \mathfrak{A}\right.
$$

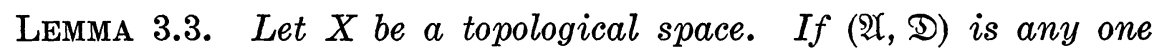

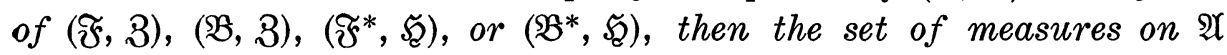
of the form $\sum_{k=1}^{n} a_{k} p_{x_{k}}$, where $a_{k} \geqq 0$ and $x_{k} \in X, k=1, \cdots, n$, is dense in $\mathfrak{M}^{+}(\mathfrak{H}, \mathfrak{D})$ with the weak topology.

The proof of [10, Th. 10, p. 187] can obviously be adapted to each of these cases.

Lemma 3.4. Let $X$ be a locally compact, Hausdorff space.

( i ) If $\nu \in \mathfrak{N}^{+}\left(\mathfrak{B}^{*}\right.$, (ㄷ), then $\nu / \mathfrak{B} \in \mathfrak{N}^{+}\left(\mathfrak{B}, \mathfrak{S}_{0}\right)$ where $\nu / \mathfrak{B}$ denotes the restriction of $\nu$ to $\mathfrak{B}$.

(ii) Suppose $\mu$ is a Baire measure in the sense of Halmos [3, p. 223] and $\nu$ denotes the unique extension of $\mu$ to a regular Borel measure in the sense of Halmos [3,54.D]. If $\mu$ is nonatomic, then so is $\nu$.

Proof. (i) Let $\mu=\nu / \mathfrak{B}$. Since $\nu$ is (5-regular, there exist $K_{i} \in \mathfrak{C}, i=1,2, \cdots$, such that $\nu\left(X-\cup_{i=1}^{\infty} K_{i}\right)=0$. Hence by [3, 50.D] there are sets $C_{i} \in \mathfrak{F}_{0}, i=1,2, \cdots$, such that, defining $B=$ $\cup_{i=1}^{\infty} C_{i}$, we have

$$
\mu(E)=\mu(E \cap B) \text { for every } E \in \mathfrak{B} .
$$

Let $\mathfrak{B}_{0}$ denote the class of Baire sets in the sense of Halmos [3, p. 220].

Since every compact $G_{\delta}$ is a zero-set, we have

$$
\mathfrak{B}_{0} \subset \mathfrak{B} \text {. }
$$

Moreover,

$$
\{E \cap B ; E \in \mathfrak{B}\} \subset \mathfrak{B}_{0}
$$

because of [1, Exercise 57.13(i)] and the fact that every zero-set is a $G_{\dot{j}}$. Since every Baire measure in the sense of Halmos is $\mathbb{C}_{0}$-regular $[3,52 . G]$, it follows easily from (1), (2), and (3) that $\mu$ is $\mathfrak{S}_{0}$-regular.

Suppose now that $\mu$ has an atom $E \in \mathfrak{B}$. Since $\nu$ is nonatomic there is a set $F \in \mathfrak{B}^{*}$ such that $F \subset E$ and $0<\nu(F)<\nu(E)$. Now there are sets $C \in \mathbb{E}, U$ open such that $C \subset F \subset U$ and $0<\nu(C) \leqq$ $\nu(F) \leqq \nu(U)<\nu(E)$ because of $\sqrt{ }$-regularity. We pick a set $C_{0} \in \mathbb{\mho}_{0}$ such that $C \subset C_{0} \subset U$ and define $H=E \cap C_{0}$. Then $H \in \mathfrak{B}$ and $H \subset E$. Moreover, $C \subset H \subset U$ so that $0<\mu(H)<\mu(E)$ which is a contradiction. 
(ii) Suppose $\nu$ has an atom $E \in \mathfrak{B}^{*}$. By the regularity of $\nu$ there is a set $C \in \mathbb{E}$ such that $C \subset E$ and $\nu(E)=\nu(C)$. As in $[1,59.1]$ we can find a set $C_{0} \in \mathfrak{F}_{0}$ such that $C \subset C_{0}$ and $\nu(C)=\nu\left(C_{0}\right)$. Now $\mu$ is nonatomic so there exists $F \in \mathfrak{B}$ such that $F \subset C_{0}$ and $0<\mu(F)<\mu\left(C_{0}\right)$. Since $C \cup F \subset C_{0}$ and $\nu(C)=\nu\left(C_{0}\right)$, we have $\nu(F-C)=0$. Consequently, if we let $H=F \cap C$, we have $H \in \mathfrak{B}^{*}, H \subset E$, and $0<\nu(F)=$ $\nu(H)<\nu(E)$ which is a contradiction.

Proof of Theorem 3.1. The proof follows closely those of Parthasarathy, Rao, and Varadhan [6, Th. 6.1] and Knowles [5, Th. 1(i)] and, in addition, makes strong use of the latter result. Let ( $\mathfrak{A}, \mathfrak{D})$

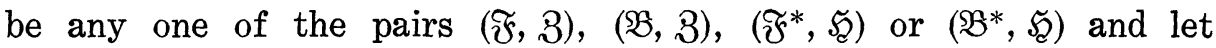
$m \in \mathfrak{M}(\mathfrak{A}, \mathfrak{D})$. We shall exhibit, for each case (but all at once), a net $\left\{m_{\alpha}\right\}$ from the appropriate $\mathfrak{N}$ space such that $m_{\alpha} \rightarrow m$ in the weak topology. Clearly we can assume $m \geqq 0$ and, moreover, that $m$ is of the form $\sum_{k=1}^{n} a_{k} p_{x_{k}}$ where $a_{k} \geqq 0$ and $x_{k} \in X, k=1, \cdots, n$ (Lemma 3.3). In any of the cases we can extend $m$ to be of this form on $\mathfrak{B}^{*}$.

Now since $X$ is locally compact, Hausdorff and perfect, it follows easily from Lemma 3.2 that the perfect, compact neighborhoods in $X$ are basic. Denote by (S) the family of $n$-vectors $\gamma=\left(U_{\gamma}\left(x_{1}\right), \cdots, U_{\gamma}\left(x_{n}\right)\right)$ where $U_{r}\left(x_{k}\right)$ is a perfect, compact neighborhood of the point $x_{k}$ and $U_{\gamma}\left(x_{k}\right) \cap U_{\gamma}\left(x_{j}\right)=\phi, j \neq k$. We direct \& by saying that $\gamma>\delta$ if, and only if, $U_{\gamma}\left(x_{k}\right) \subset U_{\delta}\left(x_{k}\right)$ for every $k=1, \cdots, n$. We fix $\gamma \in \mathbb{B}$. Since each $U_{\gamma}\left(x_{k}\right), k=1, \cdots, n$, is a perfect, compact Hausdorff space, there is by $\left[7\right.$, p. 214] or [5, Th. 1 (i)] a nonatomic, $\sqrt{ }\left(U_{\gamma}\left(x_{k}\right)\right)$-regular, countably additive Borel measure $\nu_{k}$ on $U_{\gamma}\left(x_{k}\right)$ such that $\nu_{k}\left(U_{\gamma}\left(x_{k}\right)\right)$ $=1, k=1, \cdots, n$. Define

$$
\begin{gathered}
\eta_{k}(E)=a_{k} \nu_{k}\left(E \cap U_{r}\left(x_{k}\right)\right) \text { for every } E \in \mathfrak{B}^{*}=\mathfrak{B}^{*}(X), k=1, \cdots, n, \text { and let } \\
m_{r}=\sum_{k=1}^{n} \eta_{k} .
\end{gathered}
$$

It is not hard to see that $m_{r}$ is a nonatomic, (5-regular, countably additive Borel measure on $X$ (i.e., $m_{\gamma} \in \mathfrak{N}^{+}\left(\mathfrak{B}^{*},(\mathfrak{E})\right.$ ). (Note that for any closed $K \subset X,\left\{E \cap K ; E \in \mathfrak{B}^{*}=\mathfrak{B}^{*}(X)\right\}=\mathfrak{B}^{*}(K)$, hence $\mathfrak{B}^{*}(K) \subset$ $\mathfrak{B}^{*}(X)$.) Finally, one can readily see, by elementary continuity considerations, that $m_{\gamma} \rightarrow m$ in the weak topology. Clearly this convergence also holds for the restrictions of $m_{\gamma}$ and $m$ to $\mathfrak{F}^{*}, \mathfrak{B}$, and $\mathfrak{F}$, respectively. But it follows from Lemmas 2.4 and 3.4 (i) that $m_{r} / \mathfrak{F}^{*} \in \mathfrak{N}^{+}\left(\mathfrak{F}^{*}, \mathfrak{C}\right), m_{r} / \mathfrak{B} \in \mathfrak{N}^{+}\left(\mathfrak{B}, \mathfrak{S}_{0}\right)$, and $m_{r} / \mathfrak{F} \in \mathfrak{R}^{+}\left(\mathfrak{F}, \mathfrak{C}_{0}\right)$ for every $\gamma$. The proof is complete.

COROLLARY 3.5. Let $X$ be a locally compact, Hausdorff topological space which contains a nonempty perfect subset. Then there is a nonzero, nonatomic, countably additive, (5-regular measure on $\mathfrak{F}^{*}$ 
[resp., $\left.\mathfrak{B}^{*}\right]$ and there is a nonzero, nonatomic, countably additive $\mathfrak{F}_{0^{-}}$ regular measure on $\mathfrak{F}$ [resp., $\mathfrak{B}]$.

Proof. Let $W$ denote a nonempty perfect subset of $X$. Then $\bar{W}$ (the closure of $W$ in $X$ ) is a locally compact, Hausdorff, perfect topological space in the induced topology (Lemma 3.2 (ii)) so there is a nonzero, nonatomic, countably additive, $\sqrt{ }(\bar{W})$-regular measure $\nu$ on $\mathfrak{B}^{*}(\bar{W})$ by Theorem 3.1. Hence, defining

$$
m(E)=\nu(E \cap \bar{W}) \text { for every } E \in \mathfrak{B}^{*}=\mathfrak{B}^{*}(X),
$$

we find, as before, that $m$ is a nonzero, nonatomic, countably additive, (5-regular measure on $\mathfrak{B}^{*}$. The restrictions of $m$ to $\mathfrak{F}^{*}, \mathfrak{B}$, and $\mathfrak{F}$, respectively, provide the other desired measures because of Lemmas 2.4 and 3.4 (i).

For the sake of completeness, we conclude the section by listing two converses of Corollary 3.5. They extend a result of Rudin [8, Th. 5] and, indeed, follow quite easily from it.

THEOREM 3.6. Let $X$ be a Hausdorff topological space which contains no perfect subsets. Then there is no nonzero, nonatomic, countably additive (5-regular measure on $\mathfrak{b}^{*}$ [resp., $\mathfrak{F}^{*}$ ].

Proof. Suppose there is such a measure $m$. By (5-regularity there is a compact set $K \subset X$ such that $m(K)>0$. Defining

$$
\left.\nu(E)=m(E) \text { for every } E \in \mathfrak{B}^{*}(K) \text { [resp., } \mathfrak{F}^{*}(K)\right],
$$

one readily sees that $\nu$ is a nonzero, nonatomic, countably additive $\mathfrak{F}(K)$-regular measure on $\mathfrak{B}^{*}(K)$ [resp., $\mathfrak{F}^{*}(K)$ ]. By Lemma 2.4, we can assume $\nu$ is defined on $\mathfrak{B}^{*}(K)$. Hence by a theorem of Rudin ([8, Th. 5]; [5, Th. 1 (ii)]), there is a perfect subset of $K$, hence of $X$, which is a contradiction.

REMARK. The preceding theorem remains true if we simply require our measures to be finite on compact sets; the proof goes through unscathed. This same remark applies to the next theorem.

Since any continuous function on a compact subset $K$ of a completely regular topological space $X$ can be extended to a continuous function on $X[2$, p. 43], we have $3(K)=\{E \cap K ; E \ni 3=3(X)\}$ and, consequently, similar results for $\mathfrak{F}(K)$ and $\mathfrak{B}(K)$. This fact is used in the proof of the following theorem.

THEOREM 3.7. Let $X$ be a completely regular, Hausdorff topologi- 
cal space which contains no perfect subsets. Then there is no nonzero, nonatomic, countably additive, $\mathfrak{F}_{0}$-regular measure on $\mathfrak{B}$ [resp., $\mathfrak{F}$ ].

Proof. Suppose there is such a measure $m$. There is a compact zero-set $K \subset X$ such that $m(K)>0$. Defining

$$
\nu(E)=m(E) \text { for every } E \in \mathfrak{B}(K) \text { [resp., } \mathfrak{F}(K)],
$$

we see that $\nu$ is a nonzero, nonatomic, countably additive, $\mathfrak{F}_{0}(K)$ regular measure on $\mathfrak{B}(K)$ [resp., $\mathfrak{F}(K)$ ]. By Lemma 2.4 we can assume that $\nu$ is defined on $\mathfrak{B}(K)$. Since $K$ is compact, Hausdorff, it is clear that $\nu$ is a Baire measure on $K$ in the sense of Halmos [3, p. 223]. By Lemma 3.4 (ii), $\nu$ extends to a nonzero, nonatomic, countably additive, (5-regular measure on $\mathfrak{B}^{*}$. This contradicts Theorem 3.6.

4. Concluding remarks. Clearly the hypotheses on $X$ in Theorem 3.1 and in Corollary 3.5 cannot be weakened significantly. For if $X$ is the rationals (with the relativized usual topology of the reals), then $X$ is a perfect, separable metric space. But obviously there is no nonzero, nonatomic, countably additive measure on $\mathfrak{F}, \mathfrak{B}, \mathfrak{F}^{*}$, or $\mathfrak{B}^{*}$.

\section{REFERENCES}

1. S. K. Berberian, Measure and integration, MacMillan, New York, 1965.

2. L. Gillman and M. Jerison, Rings of continuous functions, D. Van Nostrand, Princeton, New Jersey, 1960.

3. P. R. Halmos, Measure theory, D. Van Nostrand, Princeton, New Jersey, 1950.

4. R. A. Johnson, Atomic and non-atomic measures, Proc. Amer. Math. Soc. 25 \#3 (1970).

5. J. D. Knowles, On the existence of nonatomic measures, Mathematika 14 (1967), $62-67$.

6. K. R. Parthasarathy, R. Ranga Rao, and R. S. Varadhan, On the category of indecomposable distributions on topological groups, Trans. Amer. Math. Soc. 102 (1962), 200-217.

7. A. Pelczynski and Z. Semadeni, Spaces of continuous functions (III), Studia Math. 18 (1959), 211-222.

8. W. Rudin, Continuous functions on compact spaces without perfect subsets, Proc. Amer. Math. Soc. 8 (1957), 39-42.

9. A. E. Taylor, Introduction to functional analysis, Wiley, New York, 1961.

10. V. S. Varadarajan, Measures on topological spaces, (Russian) Mat. Sbornik(N.S.), (97) 55 (1961), 33-100. English translation: Amer. Math. Soc. Trans. (2) 48 (1965), 161228.

Received October 17, 1969.

WASHington State UnIVERSITY 


\title{
PACIFIC JOURNAL OF MATHEMATICS
}

\author{
EDITORS
}

\author{
H. SAMELSON \\ Stanford University \\ Stanford, California 94305 \\ RichaRd PIERCE \\ University of Washington \\ Seattle, Washington 98105
}

J. DugundJI

Department of Mathematics

University of Southern California

Los Angeles, California 9.0007

RICHARD ARENS

University of California

Los Angeles, California 9.0024

\section{ASSOCIATE EDITORS}

E. F. BeCKenBACH
B. H. NeumanN

F. WoLE

K. YoSHIDA

\section{SUPPORTING INSTITUTIONS}

\author{
UNIVERSITY OF BRITISH COLUMBIA \\ CALIFORNIA INSTITUTE OF TECHNOLOGY \\ UNIVERSITY OF CALIFORNIA \\ MONTANA STATE UNIVERSITY \\ UNIVERSITY OF NEVADA \\ NEW MEXICO STATE UNIVERSITY \\ OREGON STATE UNIVERSITY \\ UNIVERSITY OF OREGON \\ OSAKA UNIVERSITY \\ UNIVERSITY OF SOUTHERN CALIFORNIA
}

\author{
STANFORD UNIVERSITY \\ UNIVERSITY OF TOKYO \\ UNIVERSITY OF UTAH \\ WASHINGTON STATE UNIVERSITY \\ UNIVERSITY OF WASHINGTON \\ AMERICAN MATHEMATICAL SOCIETY \\ CHEVRON RESEARCH CORPORATION \\ TRW SYSTEMS \\ NAVAL WEAPONS CENTER
}

The Supporting Institutions listed above contribute to the cost of publication of this Journal, but they are not owners or publishers and have no responsibility for its content or policies.

Mathematical papers intended for publication in the Pacific Journal of Mathematics should be in typed form or offset-reproduced, (not dittoed), double spaced with large margins. Underline Greek letters in red, German in green, and script in blue. The first paragraph or two must be capable of being used separately as a synopsis of the entire paper. The editorial "we" must not be used in the synopsis, and items of the bibliography should not be cited there unless absolutely necessary, in which case they must be identified by author and Journal, rather than by item number. Manuscripts, in duplicate if possible, may be sent to any one of the four editors. Please classify according to the scheme of Math. Rev. Index to Vol. 39. All other communications to the editors should be addressed to the managing editor, Richard Arens, University of California, Los Angeles, California, 90024.

50 reprints are provided free for each article; additional copies may be obtained at cost in multiples of 50 .

The Pacific Journal of Mathematics is published monthly. Effective with Volume 16 the price per volume (3 numbers) is $\$ 8.00$; single issues, $\$ 3.00$. Special price for current issues to individual faculty members of supporting institutions and to individual members of the American Mathematical Society: $\$ 4.00$ per volume; single issues $\$ 1.50$. Back numbers are available.

Subscriptions, orders for back numbers, and changes of address should be sent to Pacific Journal of Mathematics, 103 Highland Boulevard, Berkeley, California, 94708.

PUBLISHED BY PACIFIC JOURNAL OF MATHEMATICS, A NON-PROFIT CORPORATION

Printed at Kokusai Bunken Insatsusha (International Academic Printing Co., Ltd.), 7-17, Fujimi 2-chome, Chiyoda-ku, Tokyo, Japan. 


\section{Pacific Journal of Mathematics}

\section{Vol. 34, No. $2 \quad$ June, 1970}

Shair Ahmad, On the oscillation of solutions of a class of linear fourth order

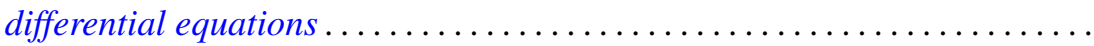

Leonard Asimow and Alan John Ellis, Facial decomposition of linearly

compact simplexes and separation of functions on cones ..............

Kirby Alan Baker and Albert Robert Stralka, Compact, distributive lattices of

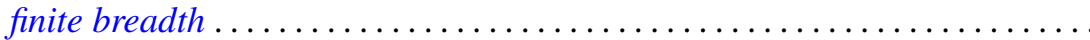

James W. Cannon, Sets which can be missed by side approximations to

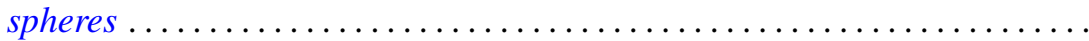

Prem Chandra, Absolute summability by Riesz means .................. 335

Francis T. Christoph, Free topological semigroups and embedding topological semigroups in topological groups....

Henry Bruce Cohen and Francis E. Sullivan, Projecting onto cycles in smooth, reflexive Banach spaces.................................

John Dauns, Power series semigroup rings .......................

Robert E. Dressler, A density which counts multiplicity ................

Kent Ralph Fuller, Primary rings and double centralizers ................

Gary Allen Gislason, On the existence question for a family of products.......

Alan Stuart Gleit, On the structure topology of simplex spaces .............

William R. Gordon and Marvin David Marcus, An analysis of equality in

certain matrix inequalities. $I \ldots \ldots \ldots \ldots \ldots \ldots \ldots \ldots$

Gerald William Johnson and David Lee Skoug, Operator-valued Feynman

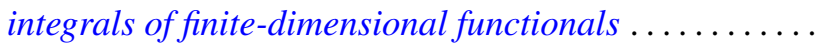

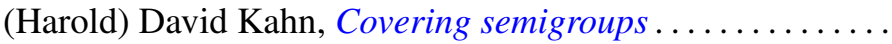

Keith Milo Kendig, Fibrations of analytic varieties

Norman Yeomans Luther, Weak denseness of nonatomic measures on perfect,

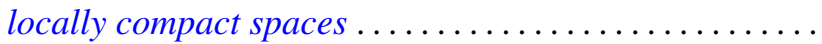

Guillermo Owen, The four-person constant-sum games; Discriminatory solutions on the main diagonal ...

Stephen Parrott, Unitary dilations for commuting contractions

Roy Martin Rakestraw, Extremal elements of the convex cone $A_{n}$ of

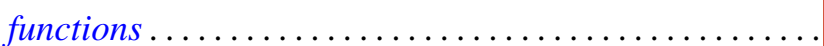

Peter Lewis Renz, Intersection representations of graphs by

William Henry Ruckle, Representation and series summability of complete

biorthogonal sequences.

F. Dennis Sentilles, The strict topology on bounded sets ...

Saharon Shelah, A note on Hanf numbers ...

Harold Simmons, The solution of a decision problem for several classes of rings. . .

Kenneth S. Williams, Finite transformation formulae involving the Legendre 